\title{
Towards Geocoding Spatial Expressions (Vision Paper)
}

\author{
Hussein S. Al-Olimat, Valerie L. Shalin, Krishnaprasad Thirunarayan and Joy Prakash Sain \\ \{hussein,valerie,tkprasad,joy\}@knoesis.org \\ Kno.e.sis Center, Wright State University, Dayton, $\mathrm{OH}$.
}

\begin{abstract}
Imprecise composite location references formed using ad hoc spatial expressions in English text makes the geocoding task challenging for both inference and evaluation. Typically such spatial expressions fill in unestablished areas with new toponyms for finer spatial referents. For example, the spatial extent of the ad hoc spatial expression "north of" or "50 minutes away from" in relation to the toponym "Dayton, $\mathrm{OH}$ " refers to an ambiguous, imprecise area, requiring translation from this qualitative representation to a quantitative one with precise semantics using systems such as WGS84. Here we highlight the challenges of geocoding such referents and propose a general formal representation that employs background knowledge, semantic approximations and rules, and fuzzy linguistic variables. We also discuss an appropriate evaluation technique for the task that is based on human contextualized and subjective judgment.
\end{abstract}

\section{CCS CONCEPTS}

- Information systems $\rightarrow$ Evaluation of retrieval results; $G e$ ographic information systems;

\section{KEYWORDS}

geocoding, spatial expressions, fuzzy spatial extents

\section{ACM Reference Format:}

Hussein S. Al-Olimat, Valerie L. Shalin, Krishnaprasad Thirunarayan and Joy Prakash Sain. 2019. Towards Geocoding Spatial Expressions (Vision Paper). In 27th ACM SIGSPATIAL International Conference on Advances in Geographic Information Systems (SIGSPATIAL '19), November 5-8, 2019, Chicago, IL, USA. ACM, New York, NY, USA, Article 4, 4 pages. https://doi.org/10.1145/3347146. 3359356

\section{INTRODUCTION}

A geographic coordinate system, such as the World Geodetic System 1984 (WGS84), quantitatively describes the exact area of named locations. However, natural language refers to a spatial area qualitatively, posing a challenge to precision in the face of the complexity of human thought process. Human qualitative spatial referents appear as both established toponyms (e.g., "Dayton, Ohio") or ad hoc spatial expressions (e.g., "north of Dayton, Ohio") linked to established/atomic toponyms with relations. In English, these often employ prepositions. The mismatch between quantitative coordinate-based referents and qualitative spatial expressions prohibits their straightforward alignment. Moreover, human spatial

Permission to make digital or hard copies of part or all of this work for personal or classroom use is granted without fee provided that copies are not made or distributed for profit or commercial advantage and that copies bear this notice and the full citation on the first page. Copyrights for third-party components of this work must be honored

For all other uses, contact the owner/author(s).

SIGSPATIAL '19, November 5-8, 2019, Chicago, IL, USA

(C) 2019 Copyright held by the owner/author(s)

ACM ISBN 978-1-4503-6909-1/19/11.

https://doi.org/10.1145/3347146.3359356 referents are not precise or formally defined, making them ambiguous, and laden with idiosyncrasies. Spatial cognition theory [11] states that "Whatever we can talk about we can also represent". However, two properties of spatial expressions challenge their representation for computing systems: they are ambiguous (requiring context) and they employ fuzzy location referents (requiring approximation). Therefore, we seek a tolerant representation, to serve as an interlingua between quantitative and qualitative spatial referents acknowledging Zadeh's incompatibility principle [19] (i.e., "the ineffectiveness of computers in dealing with human systems").

World map projects, such as OpenStreetMap, apparently solve the problem for established toponyms (e.g., cities) by representing them as polygons in GeoJSON shapefiles. Coarse-grained toponyms (aka. atomic toponyms) link to these agreed-upon quantitative representations. However, such resources are inevitably incomplete. Humans use spatial expressions to fill in the unestablished areas with new toponyms for finer spatial referents. For example, "downtown Dayton" refers to an unestablished, ad hoc portion of the larger, but atomically specified toponym (i.e., "Dayton") with imprecise and fuzzy boundaries. The number of possible mappings between this ad hoc referent and its quantitative extent, and the lack of precise and agreed upon interpretation defies both enumeration and evaluation and therein lies the complexity.

Dealing with ad hoc spatial expressions as linguistic variables [20] captures the inherent fuzziness of the referents reflecting day to day usage. While a lat/long point on a map, as in WGS84 has precise and distinctive semantics, adding linguistic expressions such as "north of" or "not far from" to that point makes it difficult for spatial systems to infer the exact spatial extent of the referent because the area is not precisely defined. This requires translation from a qualitative representation to an (imprecise) quantitative one that is adequate for the intended application and context.

In this paper, we identify and then operate on polygons to represent qualitative spatial referents. A linguistic spatial expression contrasts with polygons referenced using the WGS84 system in that such linguistic expressions come with fuzzy boundaries. This representation requires us to design heuristics to draw polygons and scale them automatically based on the context of referents (e.g., the size of the referenced toponyms [5]). The use of an ad hoc spatial expressions serves as an approximate characterization for a descriptive phrase of a location referent.

Standard evaluation methods that rely on precise boundaries are inherently incompatible with these new kinds of non-standard, but oft-used spatial referents. Precision/Recall metrics do not readily characterize the degree of error associated with referents specified with fuzzy boundaries and are missing from gazetteers. Therefore, we suggest evaluating proposed representations using a measurement scale with a higher resolution relative to a binary decision (the Likert scale), to collect human judgment and better capture the intuition behind the use of ad hoc spatial expressions. 


\section{RELATED WORK}

Vernacular Geography (VG) concerns the vagueness of location referents (aka. imprecise regions) that are not part of a gazetteer but are part of the regional culture (e.g., a "city center", "the downtown", or "the old downtown") [8]. These referents can be as big as the Midwest United States or as small as "downtown Dayton". Disagreement, between official boundaries of such locations and what people tend to consider as the boundaries, reflects contextual factors such as population density, housing systems, land use policy, and other social, cultural, and physical artifacts [8]. However, VG techniques largely have not developed formal linguistic rules and methods to deal with the semantics of location referents. Instead, these attempts are mostly deductive methods drawing on conclusions from patterns in geotagged data and textual descriptions.

Some toponym resolution techniques that map location names to their unambiguous spatial footprint rely on spatially annotated documents to learn the spatial distributions of words [6]. Usually, such supervised techniques deduce the spatial footprint of words to georeference documents based on lexical choice (e.g., "Y'all" suggests origins in the"southern US"). Woodruff and Plaunt [18] performed syntactic operations to retain relevant portions of phrases and omit others (e.g., retaining "Delta" from the phrase "literature on the Delta"). They use a relevance weighting method to assign weights to generated polygons for referents. They also support lexical transformation to improve coverage, for example, transforming "Valleys" into "Valley" that can then be found in a gazetteer. They strip tokens from toponyms, such as "County" from "Kern County", to create "evidonyms" ("Kern" in this instance), to retrieve locations like "Kern Water Bank" that is not part of the gazetteer. The technique considers the highest stacked polygons of all georeferenced locations as the focus area of a document to deduce the semantics of spatial expressions such as "south of the Delta". Lieberman and Samet [13] use geographical distances and background knowledge from gazetteers to disambiguate location references based on their relative proximity in texts. However, none of these techniques deal with fine-grained and ad hoc spatial referents in expressions that include relations such as "near" or "between".

Clementini et al. [5] analyzed qualitative distances and defined context using a three-component frame of reference (i.e., distance system, scale, and type). Crucially, the analysis shows that distance is dependent on scale. For example, the meaning of "close" in " $x$ is close to $y^{\prime \prime}$ depends on the relative position of both locations and their respective sizes. This is very useful in practice for developing heuristics to deal with spatial expressions, such as "near", that relies on the contextual toponyms in the same expression (e.g., "I live in a country near Mexico" vs. "I live in a city near Columbus, $\mathrm{OH}$ ").

Finally, in Geographic Information Retrieval (GIR) [17], the understanding of spatial relationships such as "near" in the example "beaches near Dayton" requires an understanding of the theme "beaches" and the spatial extent of the toponym "Dayton". Returning all beaches within a predefined distance, or returning the closest few beaches around the atomic toponym "Dayton", regardless of distance, are alternative ways of satisfying the query. Such approaches are limited by the availability of an entry for "beaches" in the geographic coordinate system.

\section{REFERENCING SPATIAL EXPRESSIONS}

Reference objects (aka. anchors), such as established atomic toponyms or any geocoded spatial extents (e.g., the northern side of a city), provide an initial frame of reference for ad hoc spatial referents [15]. We acknowledge that ambiguity may persist in understanding the spatial extent of atomic toponyms. However, here, we assume that atomic toponyms are correctly geocoded and disambiguated. Additionally, we assume that all atomic and ad hoc spatial referents are correctly delimited in text using techniques such as [2] and [14], and the spatial relationships are correctly annotated using techniques such as spatial role labeling [10].

The focus of this paper is to infer and geocode relative/partial spatial referents with respect to one or more identified spatial extents of anchor objects. Thus, an atomic toponym, for example, is not the head of the expression, but rather a modifier on the head, e.g., "banks" or "gas stations" in "Dayton" is more about the "banks" and "gas stations" that appear in "Dayton", than "Dayton" per se. The task then is to specify how the "Dayton" toponym enables the interpretation of "in" and the scope of the referents for "banks" and "gas stations". Landau and Jackendoff [11] suggest that representing space requires the understanding of toponyms and routes (aka. places and paths) and that prepositions extend the spatial area of a toponym to related paths and regions. The spatial relation expressed in prepositional phrases would, therefore, play the defining role of spatial extents ${ }^{1}$. In general, there are two types of spatial relations expressed in prepositional phrases:

(1) Binary spatial relations: These are the spatial referents expressed in reference to a single anchor object (e.g., "near $x$ ", "away_from $x$ ", "beside $x$ ", or "in $x "$ ).

(2) N-ary spatial relations: These are the spatial referents expressed in reference to two or more anchor objects (e.g., "near $x$ and $y$ ", "between $x$ and $y$ ", or "among $x, y$, and $z$ ").

The main frames of reference [17] that affect the interpretation of spatial extents and influence the semantics of their relations are:

(1) Relative: These are in direct relationship with the anchor objects (e.g., "in", "left_of", or "near").

(2) Absolute: This uses an external frame of reference, such as the earth's cardinal directions (e.g., "north_of" or "south_of")

(3) Intrinsic: Different from the Relative type, this adheres to the inherent properties of toponyms. For example, in "The clock tower in front of the palace", the orientation here is not important because there is only one front of the palace regardless of the relative binary relationship between the palace and the clock tower [12].

Other linguistic facts and cognitive constraints of language inform the understanding of spatial relations and prepositional phrases. In English, the encoding of subjects and objects of spatial prepositions reflects the size of objects [11]. The larger sized objects/locations always appear as the object in the case of "inside", "in", "outside", etc (e.g., for "Our office is in Ohio", the area of the "office" is much smaller than the area of "Ohio"). Similarly for adjacency relationships where larger sized spatial extents, usually with toponyms that are familiar or culturally significant, are used as a reference for smaller spatial extents, as in the example of "behind $x$ ". The use

\footnotetext{
${ }^{1}$ In this work, we do not tackle the representation of routes such as "via" and "through".
} 

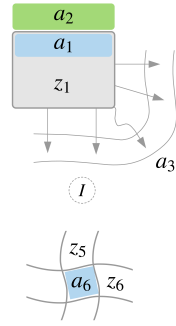

IV
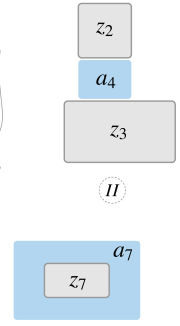

V
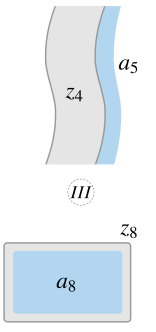

VI
Figure 1: Examples of abstract spatial representations of anchor objects $z_{(\cdot)}$ and the ad hoc referents $\mathrm{a}_{1}$ : "northern $z_{1}$ ", $\mathrm{a}_{2}$ : "north_of $z_{1}$ ", $\mathbf{a}_{3}:$ : $t$ time/distance away_from $z_{1}$ ", $\mathbf{a}_{4}$ : "between $z_{2}$ and $z_{3}$ ", a 5 : "along/on $z_{4}$ ", a 6 : " $z 5$ with $z_{6}$ ", a9: "outside/out_of $z_{7}$ ", and $\mathrm{a}_{8}$ : "in/inside $z_{8}$ ".

of "among" is interpreted as n-ary in contrast to the binary relation "between". Additionally, distances can be combined with direction markers to yield finer referents (e.g., " $5 \mathrm{~km}$ away from $x "$ ).

\section{SPATIAL REFERENTS REPRESENTATION}

Given a sentence $W=\left\langle w_{1}, w_{2}, \ldots, w_{n}\right\rangle$, let $W$ contain one or more atomic toponyms in addition to ad hoc spatial expressions defining the resolution of the referents and the extent of the focal area with spatial relations (see Section 3). While the semantics of the spatial footprint of an atomic toponym is clear (i.e., available in mapping systems and gazetteers), the spatial semantics and extent of an ad hoc expression is not clear or formally defined. Inspired by Zadeh's work on linguistic variables and values [20], we define spatial linguistic terms in ad hoc spatial expressions, such as "between" or "north_of", by defining fuzzy restrictions on their spatial extent.

Ad hoc expressions on a linguistic value (e.g., "north_of") such as " $t$ minutes" or " $m$ miles", modify (or make precise) the semantics induced by predefined syntactic rules. To represent qualitative spatial expressions, we expand the formulation of Zadeh's linguistic variable. We characterize a linguistic variable by a sextuple $(\mathcal{V}, \mathcal{I}, \mathcal{C}, \mathcal{P}, \mathcal{G}, \mathcal{S})$, where $\mathcal{V}$ is a linguistic variable (e.g., "distance"), $\mathcal{I}$ is the set of linguistic terms with fuzzy value interpretation or functional relation (e.g., "minutes_from" and "away_from"), $C$ is the set of all anchor objects, $\mathcal{P}$ is the set of spatial extents of the objects in $C, G$ is the set of spatial expression syntactic rules that generate the terms in $\mathcal{I}$, and $\mathcal{S}$ is the set of semantic rules restricting the values of each term in $\mathcal{I}$ referenced in the context of $C$.

Our intention behind using the sextuple is to enable the definition of geographic extents in a scalable manner, which is both novel and natural, and can be implemented as an iterative induction process based on the rules, semantic approximations, and background knowledge involving a single spatial relationship at a time. We regard the semantics of locative expressions not to be entirely intrinsic, it cannot be adequately captured using a conventional approach through prepositions and the propositional content, so we incorporate pragmatics, by incorporating context (especially the effect of scale) that is extrinsic to a given sentence. Moreover, cognitive constraints of the natural language from Section 3 can be employed while constructing semantic rules to further constrain the spatial extent of referents. For example, in the case of "between", the sides of the fuzzy polygon should not exceed the edge of the shortest side of the two facing polygons as we discuss next.

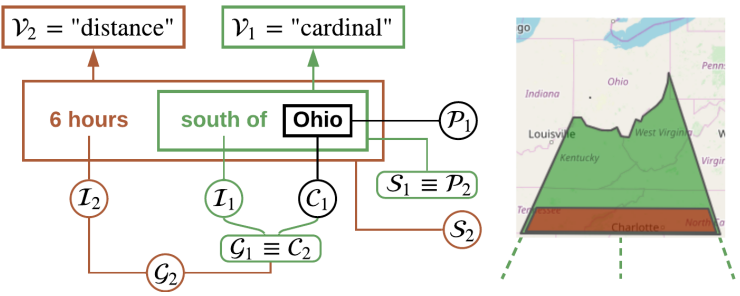

Figure 2: Spatial representation of "6 hours" and "south of" ad hoc terms in relation to the atomic toponym "Ohio".

Figure 1 visualizes spatial referents and their corresponding spatial expressions that can be formulated using our sextuple. For example, "between" (see II) can be formalized as follows:

$$
\begin{aligned}
\mathcal{V}= & \text { "topological" } \\
\mathcal{I}= & \{\text { "between"; "betwixt" }\} \\
\mathcal{C}= & \left\{c_{1}, c_{2}\right\} \\
\mathcal{P}= & \left\{c_{1} \rightarrow\left[\text { lat }_{1}, \text { long }_{1}, \text { lat }_{2}, \text { long }_{2}\right],\right. \\
& \left.c_{2} \rightarrow\left[\text { lat }_{1}, \text { long }_{1}, \text { lat }_{2}, \text { long }_{2}\right] \mid c_{1}, c_{2} \in \mathcal{C}\right\} \\
\mathcal{G}=\{ & \left\{\left\langle i, c_{1}, \text { "and", } c_{2}\right\rangle \mid(i \in \mathcal{I}) \wedge\left(c_{1}, c_{2} \in \mathcal{C}\right)\right\} \\
\mathcal{S}= & {\left[\mathcal{P}\left(c_{1}\right) \cdot \text { lat }_{2}-\alpha, \mathcal{P}\left(c_{1}\right) \cdot \text { long }_{1}-\beta,\right.} \\
& \left.\mathcal{P}\left(c_{2}\right) . \text { lat }_{1}+\alpha, \mathcal{P}\left(c_{1}\right) . \text { long }_{2}+\beta\right]
\end{aligned}
$$

where $c_{i}$ is the string name of an anchor object (e.g., "Walmart"), $\mathcal{P}$ contains the polygon for each $c_{i}$ (e.g., the simple bounding box $[38.40,-84.82,42.32,-80.51])$. Without losing generality and for the convenience of illustration and notational simplicity, we narrow down the spatial representation of each anchor object from complex polygons to rectangular form (i.e., bounding boxes) with bounds on both sides (i.e., top-right [lat $\left.1, l^{l o n g} g_{1}\right]$ and bottom-left [lat 2, long $\left._{2}\right]$ points). $\mathcal{G}$ contains only one spatial expression which describes how the spatial relationship in $\mathcal{I}$ must be used (e.g., "between Walmart and Sam's Club"). Finally, the semantics of "between" is defined as a polygon between the spatial extents of the toponyms ${ }^{2}$. As shown in Figure 1-II, the area takes the shortest facing edge of the two polygons (shown as bounding boxes) with the height as the distance between them. Therefore, the possible area of "between" can be $\mathcal{S}$, where $\operatorname{lat}_{(\cdot)}$ and $\operatorname{long}_{(\cdot)}$ are the latitudes and longitudes of the $i^{t h}$ anchor object, and $\alpha$ and $\beta$ are two offsets defined based on the semantics of $\mathcal{I}$ with $C$ to account for buffer distances [4]. ${ }^{3}$

As for the directional markers in $\langle d$, "of", $z\rangle$ where $d \in\{$ "north", $\ldots\}$, we can create a polygon representing that area in relation to the anchor object $z$ using a cone-based cardinal direction model [16]. For example, for "south of Ohio", we create a polygon $\mathcal{S}_{1}$ that sits on the bottom side of Ohio's polygon $\mathcal{P}_{1}$ (see Figure 2). The extent of $\mathcal{S}_{1}$ continues to a maximum of half of the equatorial circumference. Now, the constraint "6 hours" on the "south of Ohio" expression would create a sub-polygon $\mathcal{S}_{2}$ of the previously induced spatial extent $\mathcal{P}_{2}$ to be only the red polygon. Since "6 hours" is fuzzy, the created polygon can be padded with buffer distances to the sides of $\mathcal{S}_{2}$ equal to, for example, (a discretionary) $5 \%$ of the modifier value (i.e., \pm 18 minutes of the 6 hours constraint). Our formulation

\footnotetext{
${ }^{2}$ For more complex polygons of the two toponyms, the area inside the convex hull would make the "between" area [3]. Moreover, without further context to focus on a more precise location, all points in that induced polygon are equally likely to constitute the most precise characterization of the area in question.

${ }^{3}$ The offsets values would be different for "between" and "on" as "on" is for exterior referents with contact while "between" does not mean contact.
} 
scales automatically with the addition of more constraints and ad hoc expressions. For example, if we add "near Asheville" to the end of "6 hours south of Ohio", we would create another polygon as in Figure 2, showing the constraint on $\mathcal{S}_{2}$, in this case.

Similarly, we represent the examples in Figure 1, without loss of generality, using a complete polygon (as in $\mathbf{a}_{1}, \mathbf{a}_{2}, \mathbf{a}_{4}, \mathbf{a}_{6}$, and $\mathbf{a}_{8}$ ) or a polygon with a hole in it ( $a$ in $\mathbf{a}_{3}$ and $\mathbf{a}_{7}$ ). The challenge is not only the determination of the fuzzy boundaries for spatial extents but also the range of possible interpretations of the relative position of the polygons. In the case of $\mathbf{a}_{3}$, there are an infinite number of points that are $t$ minutes/miles away from $\mathrm{z}_{1}$, which requires more context to draw the fuzzy polygon ${ }^{4}$. Alternatively, absolute frames of reference using lexical markers such as "north_of" or "south_of" constrain the area of the referent, but without context or other lexical markers, we would draw a polygon with a hole in it covering all possible spatial extents around the atomic toponym. Finally, the alternative intrinsic frame of reference needs a full understanding of the surrounding area and requires a gazetteer with rich metadata and toponym semantics. For example, finding the front of a university campus might require searching for clusters of points of interest to suggest the orientation of the toponym. We leave further exploration and evaluation of this as future work.

\section{EVALUATION}

The majority of the techniques in the literature focus on geocoding the spatial extent of atomic toponyms (i.e., Toponym Resolution) [9]. The default evaluation metrics are Precision, Recall, and F1 as the task is to link a toponym to a gazetteer record or compare the geocoordinates with annotated toponyms in documents. Other metrics include micro and macro averaging, mean error distance, AUC of geocoding errors, and Acc@161, which calculates the accuracy of geocoding within a $161 \mathrm{~km}$ tolerance distance [7]. Clearly, such evaluation methods are incompatible with the qualitative, imprecise location referents that have no records in a gazetteer, have fuzzy meaning, and are finer than typical atomic toponyms with (sometimes) a huge error tolerance of $161 \mathrm{~km}$.

As there is no satisfactory approach in the literature or gold data available for fine-grained referents involving imprecise spatial regions, we devise an approach using a Likert scale, similar to [1] but also adding the "negative" side of the scale (i.e., including "Strongly Agree" to "Strongly Disagree"). Effectively, the individual scores ranging from 1 to -1 can be used to score all geocodings as: Score $=\frac{1}{m n} \sum_{i=0}^{n} \sum_{j=0}^{m} \sigma_{i, j}$, where $n$ is the number of spatial expressions, $m$ is the number of annotators, $\sigma$ is the weight (1 to -1 Likert scale) given to a geocoded expression by the annotator $j$, and Score is the cumulative system score to evaluate the geocoder.

\section{CONCLUSIONS AND FUTURE WORK}

In this paper, we devised a representational framework to geocode imprecise and ad hoc location referents in terms of "fuzzy" spatial extents as opposed to atomic gazetteer toponyms. Our iterative geocoding process employs rules, semantic approximations, background knowledge, and fuzzy linguistic variables. The potential disagreement between the different interpretations and the unavailability of gold standard data required us to adapt existing evaluation measures. This paper constitutes an initial step in this complex task, and we plan to continue this work by expanding on the formulation and the semantic rules, and evaluating our solution on real-world data to be annotated by multiple annotators.

\section{ACKNOWLEDGMENTS}

This research was supported by the NSF award EAR-1520870 "Hazards SEES: Social and Physical Sensing Enabled Decision Support for Disaster Management and Response". All views are those of the authors and do not necessarily reflect the views of the sponsor.

\section{REFERENCES}

[1] Niloofar Aflaki, Shaun Russell, and Kristin Stock. 2018. Challenges in Creating an Annotated Set of Geospatial Natural Language Descriptions (Short Paper). In GIScience 2018. Schloss Dagstuhl-Leibniz-Zentrum fuer Informatik.

[2] Hussein S. Al-Olimat, Krishnaprasad Thirunarayan, Valerie Shalin, and Amit Sheth. 2018. Location Name Extraction from Targeted Text Streams using Gazetteer-based Statistical Language Models. In Proceedings of the 27th International Conference on Computational Linguistics. Association for Computational Linguistics, 1986-1997.

[3] Roland Billen and Eliseo Clementini. 2004. A model for ternary projective relations between regions. In International Conference on Extending Database Technology. Springer, 310-328.

[4] Hao Chen, Stephan Winter, and Maria Vasardani. 2018. Georeferencing places from collective human descriptions using place graphs. Journal of Spatial Information Science 2018, 17 (2018), 31-62.

[5] Eliseo Clementini, Paolino Di Felice, and Daniel Hernandez. 1997. Qualitative representation of positional information. Artif. Intel. 95, 2 (1997), 317 - 356.

[6] Grant DeLozier, Jason Baldridge, and Loretta London. 2015. Gazetteerindependent Toponym Resolution Using Geographic Word Profiles. In AAAI'15. AAAI Press, 2382-2388.

[7] Milan Gritta, Mohammad Taher Pilehvar, and Nigel Collier. 2018. A Pragmatic Guide to Geoparsing Evaluation. arXiv preprint arXiv:1810.12368 (2018).

[8] Livia Hollenstein and Ross Purves. 2010. Exploring place through user-generated content: Using Flickr tags to describe city cores. Fournal of Spatial Information Science 2010, 1 (2010), 21-48.

[9] Ehsan Kamalloo and Davood Rafiei. 2018. A Coherent Unsupervised Model for Toponym Resolution. In Proceedings of (WWW'18). Republic and Canton of Geneva, Switzerland, 1287-1296. https://doi.org/10.1145/3178876.3186027

[10] Parisa Kordjamshidi, Martijn Van Otterlo, and Marie-Francine Moens. 2010. Spatial Role Labeling: Task Definition and Annotation Scheme.. In LREC.

[11] Barbara Landau and Ray Jackendoff. 1993. "What" and "where" in spatial language and spatial cognition. Behavioral and Brain Sciences 16, 2 (1993), 217-238.

[12] Stephen C Levinson and Stephen C Levinson. 2003. Space in language and cognition: Explorations in cognitive diversity. Vol. 5. Cambridge University Press.

[13] Michael D. Lieberman and Hanan Samet. 2012. Adaptive Context Features for Toponym Resolution in Streaming News. In Proceedings of the (SIGIR '12). ACM, New York, NY, USA, 731-740. https://doi.org/10.1145/2348283.2348381

[14] Fei Liu, Maria Vasardani, and Timothy Baldwin. 2014. Automatic identification of locative expressions from social media text: A comparative analysis. In Proceedings of the 4th Int. Workshop on Location and the Web. ACM, 9-16.

[15] Yu Liu, Qing H Guo, John Wieczorek, and Michael F Goodchild. 2009. Positioning localities based on spatial assertions. International fournal of Geographical Information Science 23, 11 (2009), 1471-1501.

[16] Yu Liu, Xiaoming Wang, Xin Jin, and Lun Wu. 2005. On internal cardinal direction relations. In Int. Conference on Spatial Information Theory. Springer, 283-299.

[17] Ross S Purves, Paul Clough, et al. 2018. Geographic Information Retrieval: Progress and Challenges in Spatial Search of Text. Foundations and Trends ${ }^{\circledR}$ in Information Retrieval 12, 2-3 (2018), 164-318.

[18] Allison Gyle Woodruff and Christian Plaunt. 1994. Gipsy: Georeferenced information processing system,". Journal of the American Society for Information Science 45, 9 (1994), 645-655.

[19] L. A. Zadeh. 1973. Outline of a New Approach to the Analysis of Complex Systems and Decision Processes. IEEE Transactions on Systems, Man, and Cybernetics SMC3, 1 (Jan 1973), 28-44. https://doi.org/10.1109/TSMC.1973.5408575

[20] Lotfi Asker Zadeh. 1975. The concept of a linguistic variable and its application to approximate reasoning-I. Information sciences 8, 3 (1975), 199-249.

\footnotetext{
4 "As the crow flies" method or route-based distance calculation can be used assuming "cars" as the culturally determined mode of transportation.
} 